Dom. Cien., ISSN: 2477-8818

Vol. 5, núm.1., ene, 2019, pp. 603-623

\title{
Lesiones de la mucosa oral asociadas al uso de prótesis odontológicas en pacientes edéntulos totales
}

\section{Oral mucosal lesions associated with the use of dental prostheses in total edentulous patients}

\section{Lesões da mucosa bucal associadas ao uso de prótese dentária em pacientes totalmente desdentados}

\author{
Manuel A. León-Velastegui ${ }^{\text {I }}$ \\ maleon@unach.edu.ec \\ Natalia A. Gavilanes-Bayas II \\ natalia.gavilanes@unach.edu.ec \\ Edwin X. Mejía-Navas ${ }^{\text {III }}$ \\ exaby_mn@hotmail.com
}

Recibido: 27 de noviembre de 2018 * Corregido: 18 de diciembre de 2018 * Aceptado: 20 de diciembre de 2018

I. Especialista en Rehabilitación Oral; Diploma Superior en Odontología Estética; Odontólogo; Docente Facultad de Ciencias de la Salud, Carrera de Odontología, Universidad Nacional del Chimborazo; Riobamba, Ecuador.

II. Especialista en Rehabilitación Oral; Odontóloga; Docente Facultad de Ciencias de la Salud, Carrera de Odontología, Universidad Nacional del Chimborazo; Riobamba, Ecuador.

III. Odontólogo, Facultad de Ciencias de la Salud, Carrera de Odontología, Universidad Nacional del Chimborazo; Riobamba, Ecuador. 


\title{
Resumen
}

El presente trabajo de investigación tuvo como objetivo determinar las lesiones de la mucosa oral asociadas al uso de prótesis odontológica en pacientes edéntulos totales, mediante una revisión bibliográfica, realizada a través de una investigación de tipo explicativa y documental, sobre una población conformada por 219.503 artículos científicos publicados en revistas especializadas e indexadas de tipo internacional durante el período 2008-2018. Se seleccionó una muestra por conveniencia 35 publicaciones registradas en las base de datos PUBMED (14 artículos científicos), Google académico (8 publicaciones) y Redalyc (13 artículos) que alcanzaron los criterios de selección del trabajo de investigación. Lo que permitió determinar que las principales lesiones de la mucosa oral asociadas al uso de la prótesis en pacientes edéntulos totales son la estomatitis protésica $(71,43 \%)$, la úlcera traumática $(28,57 \%)$ y la hiperplasia fibrosa $(54,29 \%)$, siendo la estomatitis protésica la de mayor prevalencia debido a un conjunto de factores como son la deficiente higiene oral $(17,14 \%)$, prótesis completa inestable $(14,29 \%)$ y de uso prolongado $(14,29 \%)$, siendo el género femenino el más afectado por este tipo de lesiones (65,71\%), así como pacientes con edad superior a los 70 años $(22,86 \%)$.

Palabras claves: Lesiones de la Mucosa Oral; Prótesis Odontológicas; Edentulismo Total; Pacientes Edéntulos.

\begin{abstract}
The present research work aimed to determine the oral mucosal lesions associated with the use of dental prostheses in total edentulous patients, through a literature review, carried out through an explanatory and documentary type of research, on a population made up of 219,503 scientific articles published in specialized and indexed international journals during the period 2008-2018. A convenience sample was selected 35 publications registered in the PUBMED database (14 scientific articles), Google academic (8 publications) and Redalyc (13 articles) that met the selection criteria of the research work. This allowed determining that the main lesions of the oral mucosa associated with the use of the prosthesis in total edentulous patients are prosthetic stomatitis (71.43\%), traumatic ulcer $(28.57 \%)$ and fibrous hyperplasia (54,29\%), prosthetic stomatitis being the most prevalent due to a set of factors such as poor oral hygiene (17.14\%), complete unstable prosthesis
\end{abstract}


(14.29\%) and prolonged use (14.29\%), being the female gender the most affected by this type of lesions $(65.71 \%)$, as well as patients older than 70 years $(22.86 \%)$.

Key words: Oral Mucosal Lesions; Dental Prostheses; Total Edentulism; Edentulous Patients.

\section{Resumo}

O presente trabalho teve como objetivo determinar as lesões da mucosa bucal associadas ao uso de prótese dentária em pacientes totalmente desdentados, por meio de uma revisão de literatura, realizada por meio de pesquisa explicativa e documental, sobre uma população composta por 219.503 artigos científicos publicados em revistas internacionais especializadas e indexadas durante o período 2008-2018. Uma amostra de conveniência foi selecionada 35 publicações cadastradas na base de dados PUBMED (14 artigos científicos), Google acadêmica (8 publicações) e Redalyc (13 artigos) que preencheram os critérios de seleção do trabalho de pesquisa. Isso permitiu determinar que as principais lesões da mucosa bucal associadas ao uso da prótese em pacientes totalmente edêntulos são estomatite protética $(71,43 \%)$, úlcera traumática $(28,57 \%)$ e hiperplasia fibrosa $(54,29 \%)$, sendo a estomatite protética mais prevalente devido a um conjunto de fatores como má higiene bucal $(17,14 \%)$, prótese instável completa $(14,29 \%)$ e uso prolongado $(14,29 \%)$, sendo o sexo feminino o mais acometido por esse tipo de lesão $(65,71 \%)$, bem como pacientes com mais de $70 \operatorname{anos}(22,86 \%)$.

Palavras-Chave: Lesões Mucosas Bucais; Próteses Dentárias; Edentulismo Total; Pacientes desdentados.

\section{Introducción.}

La pérdida de piezas dentales es el resultado principalmente de las caries y las patologías periodontales, aunque también depende de la actitud del paciente, del profesional odontólogo, la viabilidad, accesibilidad a la atención odontológica y a los estándares de cuidado. Estos factores producen con gran frecuencia edentulismo, bien sea parcial o total, que corresponde al estado dental más prevalente en la población de mayor edad, ocasionando un grado variable de invalidez o 
incapacidad oral, el cual solamente puede mejorarse mediante una óptima rehabilitación protésica.

El restablecimiento de las piezas dentales extraídas, mediante prótesis destituibles o fijas beneficia diferentes funciones fisiológicas; como por ejemplo, la fonación, deglución, masticación y renovación de la estética perdida. Aun cuando esta alternativa promete ser beneficiosa no se encuentra exenta de propiciar daños relacionados con la susceptibilidad a los tejidos, derivados de incorrectos estilos de vida o por el envejecimiento que producen trasformaciones importantes en las mucosas y huesos de la cavidad oral; pero sobretodo, que dan paso a la aparición de las lesiones. (1)

La presencia de lesiones de la mucosa oral afecta el estado de salud, general, de las personas. Esto se debe a que son patologías acumulativas o progresivas que propician trastornos fisiológicos muy complejos que pueden llegar a afectar el estilo de comer, la forma de comunicarse, el aspecto, en ocasiones, producen dolor, molestias. El área más frecuente para el desarrollo de las lesiones es la zona que mantiene el contacto constante con la parte interna de la prótesis. Esto se debe a que este punto se convierte, por la mala práctica de higiene, a la presencia de microorganismos oportunistas aglutinados a las partes de menor acceso a la limpieza donde predomina el contacto directo con las mucosas; entre las que se pueden citar: paladar, suelo de boca, reborde alveolar, comisura labial, entre otras. (1)

Estas lesiones paraprotésicas pueden diagnosticarse de dos tipos: agudas y crónicas. La primera suele ser producto del uso de prótesis nuevas mal ajustadas que ejercitan una presión enorme contra los tejidos blandos lo que favorece la aparición de ulceraciones o isquemia arterial y/o dolor. La segunda, son el resultado de prótesis inestables que producen roces sobre el tejido lo que estimulan modificaciones graduales del tejido de sostén. Sin duda, la opción de cubrir las zonas de soporte con 
prótesis representa un ataque mecánico no fisiológico para los tejidos que pueden ser víctimas de irritación tisular, reacciones inflamatorias. (2)

El diseño, fabricación y aplicación de cualquier prótesis no solamente tiene como objetivo básico reponer piezas dentales perdidas y tejidos subyacentes para una mejor estética y funcionamiento, sino también para mejorar los tejidos permanentes mediante una concepción bien clara de los estados de reposo y actividad de la estructura dental con precaución de no ocasionar daños a dichos tejidos. (3)

Un estudio realizado en el Líbano por El Toum (2) señala que los estudios epidemiológicos acerca de las lesiones de la mucosa oral son escasos en comparación con la caries y las patologías periodontales, variando de manera significativa la prevalencia entre diversos países y áreas desde un 4,9\% hasta un 64,7\%. Asimismo, aclara de Oliveira (4), en la investigación realizada en Brasil que de la prevalencia mundial de las lesiones de mucosa oral, aproximadamente el $29 \%$ se encuentran relacionadas con el uso de prótesis dental, existiendo una fuerte asociación entre el uso prolongado del dispositivo y la presencia de lesiones orales.

Es por ello, que la Organización Mundial de la Salud (OMS) en 2005 (5), presentó una clasificación específica sobre las lesiones de mucosa oral, especialmente las potencialmente malignas, mencionando la leucoplasia y la eritroplasia. Además, de otras lesiones como la fibrosis submucosa bucal y el liquen plano entre las alteraciones latentemente malignas (6).

En el Ecuador, un estudio desarrollado por Espinosa en 2014 (7), con el objetivo de determinar las principales alteraciones en la mucosa oral en pacientes geriátricos portadores de prótesis dental, evidenciando mediante la evaluación clínica a la cavidad oral que el 69\% de la población afectada 
corresponde al género femenino, muestran modificaciones en la mucosa, lo que ha sido asociado con la presencia de prótesis total. Por otro lado, el 31\% presenta alteraciones por el uso de prótesis removible.

En la investigación de Mayorga 2017(8), aplicada a la población de Riobamba, con la finalidad de determinar la incidencia de las principales patologías orales en pacientes geriátricos, los cuales se caracterizan por presentar edentulismo parcial (53\%) o total (47\%) y uso de prótesis dental, se determinó mediante análisis clínico que entre las principales patologías localizadas en la mucosa oral se encontraba la estomatitis protésica (23\%), generalmente causada por prótesis desajustadas, mala higiene de los aparatos e infecciones bacterianas alojadas debajo de los mismos.

Estos resultados son atribuidos a factores predisponentes tales como la candidiasis, conocida como la infección micótica de afectación oral más frecuente causada por el crecimiento exagerado de las colonias de Candida albicans, el cual es un hongo diploide asexual que forma parte del ecosistema bacterial habitual presente en la cavidad bucal (9), además de problemas mecánicos y el uso prolongado de prótesis, así como el área mayor de la mucosa oral cubierta por una prótesis total removible, detectándose también que las premalignidades y tumores malignos se incrementan en la medida que la población envejece. (10)

Por lo tanto, la presente investigación es importante porque se determinó las lesiones de la mucosa bucal prevalentes, describiendo los factores relacionados con el uso de prótesis en pacientes edéntulos totales, tales como el estado de conservación de la prótesis, tiempo de uso y hábitos de higiene deficientes, entre las causas principales de la aparición y desarrollo de este tipo de patologías (8). El estudio determinó los diversos tipos de lesiones asociadas al uso de prótesis dental en pacientes edéntulos totales, mediante la recopilación de información de interés proporcionando 
beneficios a los profesionales de Odontología, estudiantes de la Carrera de Odontología de la Universidad Nacional de Chimborazo y a los pacientes edéntulos totales que utilizan prótesis dentales, debido al aporte de información fundamental para el adecuado uso de la prótesis a fin de mejorar los tratamientos aplicados, permitiendo también diseñar e implementar medidas correctivas y preventivas que optimicen el nivel de salud oral y en consecuencia mejoren la salud en general del pacientes. Por lo antes descrito es que plantea el siguiente objetivo: Determinar las lesiones de la mucosa oral asociadas al uso de prótesis odontológica en pacientes edéntulos totales a partir de una revisión bibliográfica.

\section{Materiales y métodos.}

\section{Tipo de estudio}

El tipo de estudio es explicativa y documental, que se realizó en función de una revisión bibliográfica relacionada con las lesiones de la mucosa oral asociado al uso de la prótesis en pacientes edéntulos totales, a nivel mundial en el período 2008-2018, este estudio servirá de precedente para que los investigadores puedan tener un medio de consulta confiable sobre el tema. La recolección de datos se realizó de fuentes bibliográficas (11), artículos científicos de PUBMED (www.pubmed.gov), Google académico (www.scholar.google.es), Redalyc (www.redalyc.org).

\section{Universo y unidades de estudio}

La población estuvo conformada en su totalidad por 219.503 artículos científicos publicados en revistas especializadas e indexadas publicados durante el período 2008-2018, los que consideraron temáticas sobre las lesiones de la mucosa oral asociado al uso de la prótesis odontológica en pacientes edéntulos totales. En la selección de la muestras se utilizó el muestreo no probabilístico 
por conveniencia (11), de la población de artículos científicos durante el período 2008-2018, sobre lesiones de la mucosa oral, se eligieron 35 publicaciones de las lesiones de la mucosa oral asociadas al uso de la prótesis odontológica en pacientes edéntulos totales, registradas en las base de datos de PUBMED (14 artículos científicos), Google académico (8 publicaciones) y Redalyc (13 artículos), y que cumplan con los siguientes criterios de inclusión y exclusión de la investigación:

\section{Procedimientos y Técnicas}

Para cumplir con los objetivos la investigación se dividió en tres fases:

\section{Fase I. Búsqueda de información}

Inicialmente se buscó artículos científicos de revistas indexadas tanto de español como en inglés sobre las lesiones de la mucosa oral asociado al uso de la prótesis odontológicas en pacientes edéntulos totales, durante el período de 2008-2018, para lo cual se seleccionaron los buscadores más confiables de la web en el área de odontología, PUBMED (www.pubmed.gov), Google académico (www. scholar.google.es), Redalyc (www.redalyc.org), estructurándose en función de las variables de estudio y el tiempo 2008-2018.

\section{Fase II. Organización de la información}

De la información obtenida de la búsqueda de bibliográfica se seleccionó la más relevante, pertinente sobre el tema, que cumplían con los criterios de selección, enfocándose en las lesiones de la mucosa oral asociado al uso de la prótesis dentales en pacientes edéntulos totales, también se utilizaron programas especiales de libre acceso como Mendeley Desktop, con lo cual fue más fácil la sistematización de la información. Para lo cual, se usó una estructura organizativa mediante tablas 
de datos, tomando en cuenta las variables, autor, año de publicación, buscador web, país, metodología y documentación de gran importancia para el desarrollo de la investigación.

\section{Fase III. Análisis de los datos e información}

En esta fase se identificó que aporte se puede lograr con los datos recopilados, los resultados sobre las lesiones de la mucosa oral asociado al uso de la prótesis odontológicas en pacientes edéntulos totales, de estudios de impacto mundial, en función de las causas, consecuencias, género y edad de los pacientes afectados por las lesiones de la mucosa oral, logrando con esto cumplir con los objetivos planteados en la investigación. Fue necesario con los resultados de los estudios previos realizar figuras, tablas comparativas, con valores cuantitativos, los cuales se plasmaron en una hoja del Programa Microsoft Excel, para hacer las representaciones gráficas correspondientes, que fueron de utilidad para llegar a las conclusiones del estudio. Por ser una investigación totalmente documental, no requirió ser evaluado por un comité de ética de la UNACH. Solo se efectuó una búsqueda por la web de artículos sobre el tema, sin la intervención de pacientes o estudio in vitro.

\section{Resultados y discusión.}

Las lesiones de la mucosa oral comprenden una gran variedad de alteraciones diversas localizadas en el tejido blando de la cavidad oral, puede verse afectado por una gran variedad de lesiones y afecciones, algunas de las cuales son inofensivas, mientras que otras pueden presentar complicaciones graves. La identificación y el tratamiento de estas patologías son una parte importante del cuidado de la salud oral total. (12-14)

De acuerdo Turker (13), menciona que los adultos mayores portadores de prótesis completa son propensos a presentar lesiones de la mucosa oral, desde reacciones agudas a crónicas, resultados que 
se encuentran directamente relacionados con la utilización de prótesis. Las afecciones inflamatorias agudas y crónicas de la mucosa oral pueden clasificarse en función de los respectivos factores etiológicos. $(14,15)$

Es relevante entender que la prótesis es un cuerpo extraño en la cavidad oral, que provoca inestabilidad y variación en el área bucal, que ocasiona que los tejidos se readapten a este objeto, que dependen de la reacción de cada organismo. Uno de los grandes problemas es el uso constante y desajuste del aparato protésico, más otros factores como la precaria higiene de la boca y de la prótesis inducen a que se produzca las lesiones de la mucosa oral, que desmejoran las condiciones de la salud bucal de los pacientes. $(14,16,17)$

\section{Determinación de las Lesiones de la Mucosa Oral asociadas al uso de prótesis odontológica en pacientes Edéntulos Totales}

Las lesiones de la mucosa oral en edéntulos totales portadores de prótesis varían entre un rango de $62,9 \%$ al $69 \%$, de acuerdo a la literatura las lesiones orales asociadas por el uso de prótesis incluyen estomatitis protésica, candidiasis eritematosa, queilitis angular, queratosis traumática e hiperplasia papilar (17-19). A continuación se detallan los tipos de lesiones más frecuentes:

Según los resultados de la figura 1, se detectaron en 25 artículos científicos que la principal lesión de la mucosa oral es la estomatitis protésica (71,43\%), de acuerdo Matos (20) la estomatitis subprotésica es una inflamación crónica de la mucosa oral del paladar consecuencia del contacto de ésta con una prótesis, perjudicando, esencialmente, a las personas de avanzada edad. $(1,21)$

Atashrazm y Sadri (22), la estomatitis dental se define como eritema localizado o difuso que afecta a una parte de la mucosa recubierta o como una lesión inflamatoria papilar o granular que incide en la 
parte central del paladar duro y la cresta alveolar. Es un trastorno común que afecta a los usuarios de prótesis, se caracteriza como inflamación y eritema de las áreas de la mucosa oral cubiertas por el aparato protésico. A pesar de lo que es común, la etiología de la estomatitis por prótesis dental no se comprende por completo. $(21,23)$

Según Zwiri (18), es una enfermedad infecciosa en la que la mucosa oral por bacterias en el área debajo de la dentadura; es probable que ocurran úlceras debido a prótesis recién colocadas y a la reabsorción del hueso alveolar. De acuerdo a Marín (24) y García (17), la prevalencia de estomatitis protésica se encuentra afecta alrededor del 30 al 50\% de los portadores de aparato protésico, es por esta razón que la mayoría de las publicaciones lo atribuye como la principal lesión de la mucosa oral asociado al uso de la prótesis en pacientes edéntulos totales.

\section{Figura 1. Primera lesión de la mucosa oral}

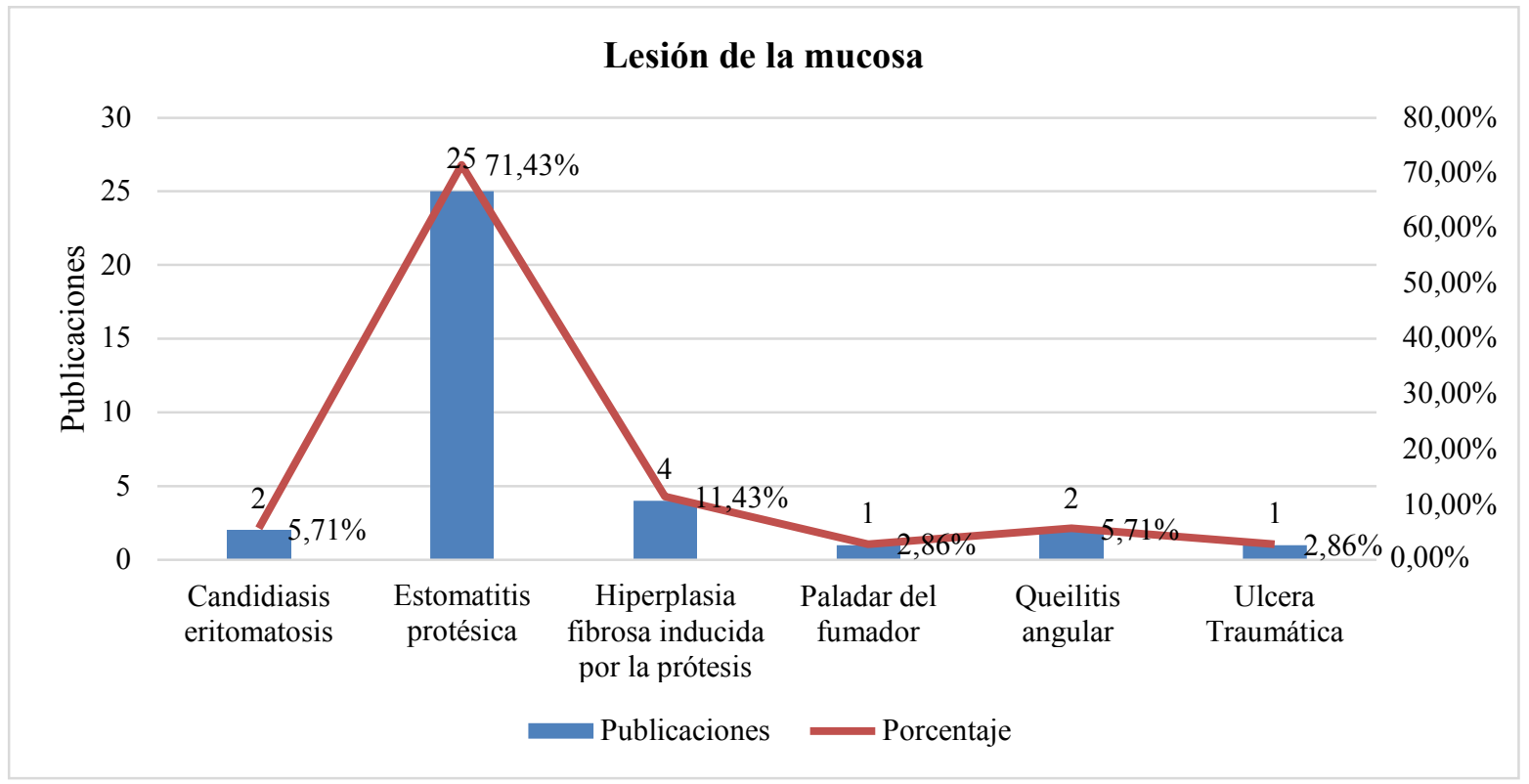

\section{Identificación de las principales causas de Lesiones de la Mucosa Oral que se encuentran relacionadas al uso de la prótesis en pacientes Edéntulos Totales}


Los traumatismos crónicos de larga duración pueden ser causal de lesiones de la mucosa oral, por efecto de la prótesis, como las superficies basales de prótesis dentales mal adaptadas, el tiempo de uso, mal estado del aparato protésico (quebradas), mal higiene oral y de la prótesis, dimensiones verticales inadecuadas y confeccionadas por profesionales inhabilitados. $(17,25,26)$

Para Lemus (14), existen contradicciones sobre los factores asociados a las lesiones de la mucosa oral y la forma en que actúan para causar esta patología, menciona que entre los factores o causas locales son los traumáticos por la presencia de la prótesis, la sequedad bucal y la higiene oral (25), explica que dentro de la literatura se encuentra ausente un consenso sobre algunos elementos importantes; como la calidad y características de la prótesis. (23)

La primera causa de la lesión de la mucosa oral de los edéntulos totales es por efecto del uso prolongado de la prótesis $(22,86 \%)$, prótesis completa inestables $(20,00 \%)$ y la deficiente higiene bucal $(20,00 \%)$ (Figura 2).

Estos hallazgos son confirmados por Lemus (14), quienes manifiestan que los edéntulos totales, usan por tiempo prolongado la prótesis, que no se la quitan por la noche, lo que produce la lesión de la mucosa oral por causas mecánica, debido a que la prótesis se comporta como un factor traumático local, que al estar en contacto con la mucosa más allá del tiempo recomendado al día, irrita la mucosa, aunado a la deficiente higiene oral, se presentan cambios fisiológicos que se convierten con el tiempo en lesiones del tejido blando. $(17,27)$

Figura 2. Principal causa de la lesión de la mucosa oral 


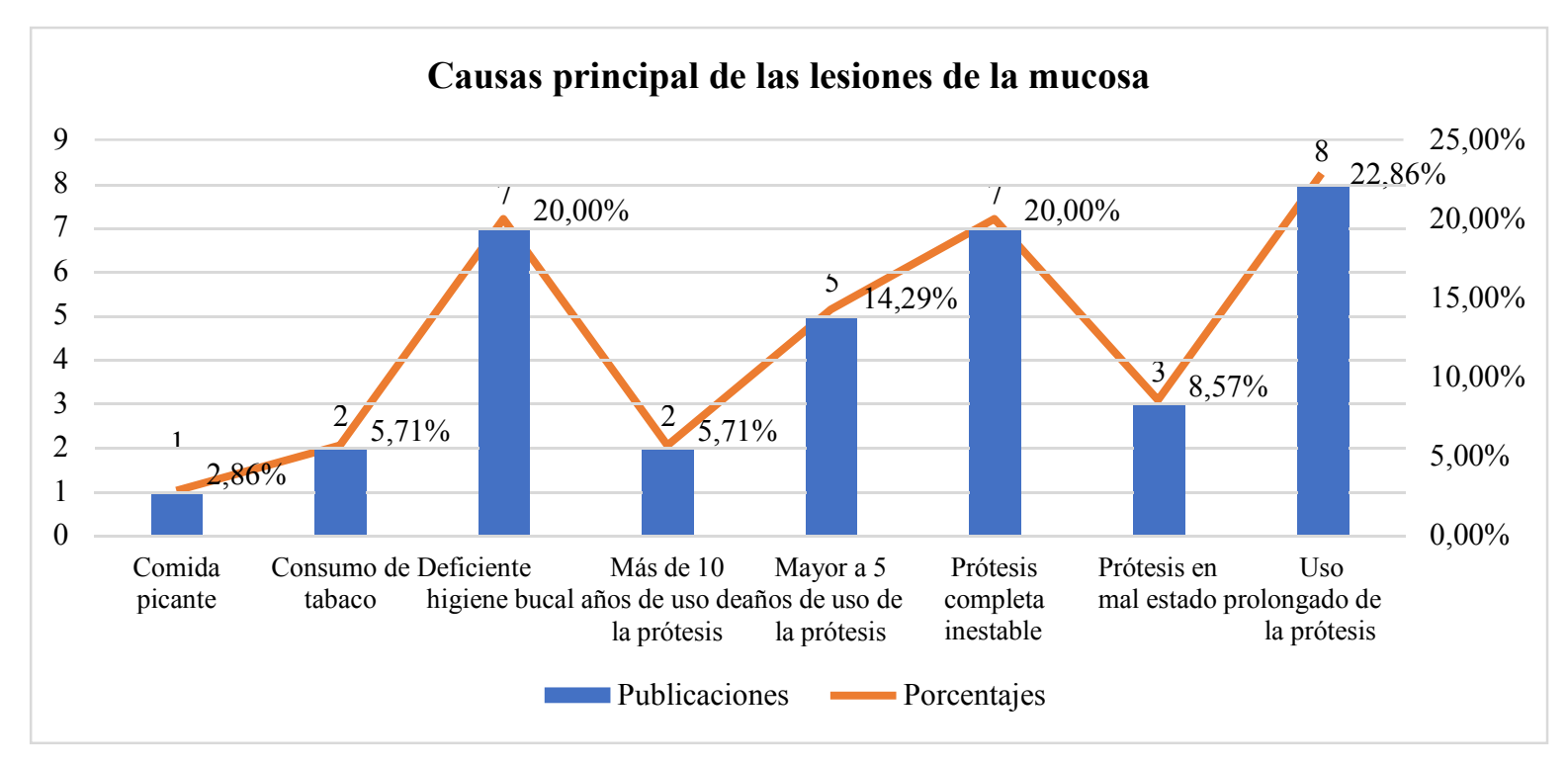

\section{Causas de las lesiones}

Sobre la etiología de las lesiones de la mucosa oral en edéntulos totales, son muy variadas, pero principalmente se encuentra el hecho que el paciente usa el aparato protésico 10 años o más, las 24 horas al día, la precaria higiene bucal y de la prótesis, alergias, problemas inmunológico, mala calidad del material protésico, el avance de la edad y el sexo, estos son considerados los factores de riesgo para las lesiones orales. $(3,27,28)$

De acuerdo a Lira (29), existe tres veces más probabilidad de que los edéntulos totales presenten una o más lesiones de la mucosa oral, por efecto del uso de prótesis que ocurren que tienen lugar, en más del 50\% de los diagnósticos. $(17,27)$

La principal lesión de la mucosa oral asociados al uso de la prótesis odontológica en pacientes edéntulos totales, es la estomatitis protésica, que de acuerdo a 6 publicaciones sobre el tema, la causa es la deficiente higiene oral, seguido por prótesis completa inestable y uso continuo de la prótesis. 
Según Mandali (28), la estomatitis protésica es un proceso inflamatorio de la mucosa oral asociada con la presencia de Cándida u otros microorganismos, por el uso de prótesis dentales, pH salival ácido, ingestión alta de carbohidratos, terapia antibiótica a largo plazo, terapia hormonal, diabetes mellitus o hipertensión arterial que generan consecuencias directas en el contexto de la cavidad bucal. La irritación mecánica de la prótesis y la disminución de la destreza manual para la higiene oral por parte de pacientes geriátricos predisponen a la población desdentada a la estomatitis protésica. $(25,30)$

\section{Descripción de las consecuencias de las Lesiones de la Mucosa Oral asociadas al uso de prótesis en pacientes Edéntulos Totales}

Con respecto a las consecuencias de las lesiones de la mucosa oral, solo 14 autores describieron las consecuencias para los edéntulos totales, como el daño en el paladar duro, dolor, inflamación y lesiones pre malignas (Figura 3).

Sobre esto Marín (24), que por el uso constante de la prótesis, los edéntulos totales presentan como consecuencia afección de la zona del paladar duro, debido a las fuerzas de compresión y tracción que se genera del contacto de la prótesis con esa región de la anatomía bucal.(23,31) 


\section{Figura 3. Consecuencias}

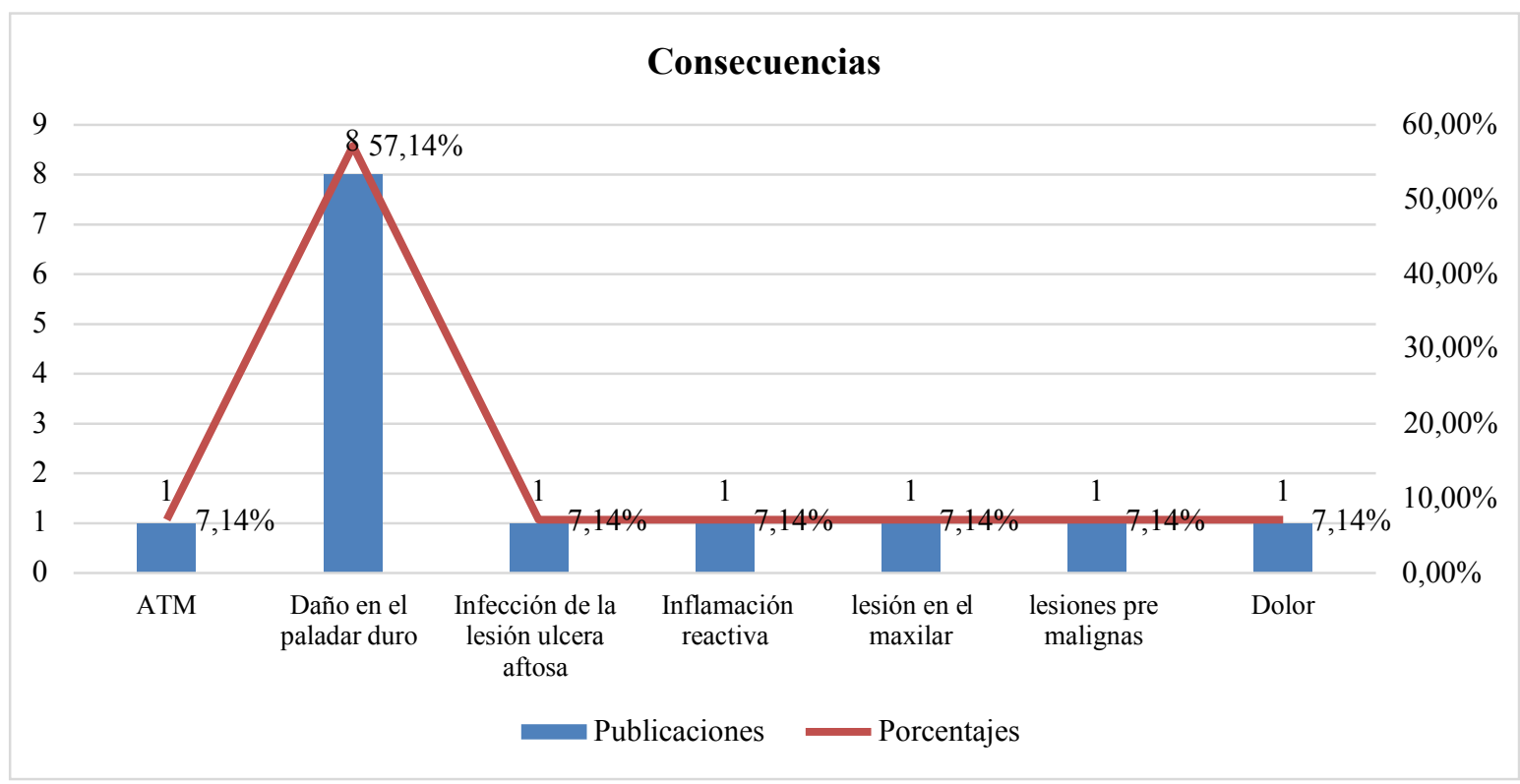

\section{Caracterización de las Lesiones de la Mucosa Oral asociadas al uso de prótesis odontológica en}

\section{función del Sexo y la Edad de los pacientes Edéntulos Totales}

\section{- Sexo de los edéntulos totales afectados por lesiones de la mucosa oral}

Gran número de investigaciones, señalan que el sexo más propenso a presentar lesiones de la mucosa oral, relacionada con la utilización de prótesis odontológica en pacientes edéntulos totales, es el femenino $(65,71 \%)$ y en menor proporción el masculino, tal como se evidencia en la figura 4.

Estos datos son corroborados por Gendreau y Loewy (21), quienes aducen que en general, la incidencia de las lesiones de la mucosa oral es más alta entre los usuarios de prótesis dentales de edad avanzada y entre las mujeres, por causas del uso continuo del aparato protésico, cambios hormonales, además son las que visitan frecuentemente al odontólogo, en comparación con los hombres. $(14,27)$ 
Gaur (19) la mayor frecuencia de lesiones inducidas por la dentadura entre mujeres no se comprende mucho, pero puede explicarse por razones hormonales y relacionadas con la edad. En el caso de mujeres perimenopáusicas y posmenopáusicas, la disminución del estrógeno y la progesterona y la atrofia de la mucosa oral pueden contribuir a la exacerbación de la respuesta inflamatoria por irritación crónica causada por el uso de prótesis completa, lo que aumenta la incidencia de la mucosa oral lesiones en pacientes mujeres. $(21,27)$

Sin embargo, Marín (32), determinó que existe mayor cantidad de hombres afectados por lesiones de la mucosa oral asociado al uso de prótesis completa que mujeres, aducen que esto se debe a las diferencias fisiológicas, a las funciones o roles en la sociedad de cada sexo. (33)

\section{Figura 4. Sexo de los pacientes afectado por la lesión de la mucosa oral}

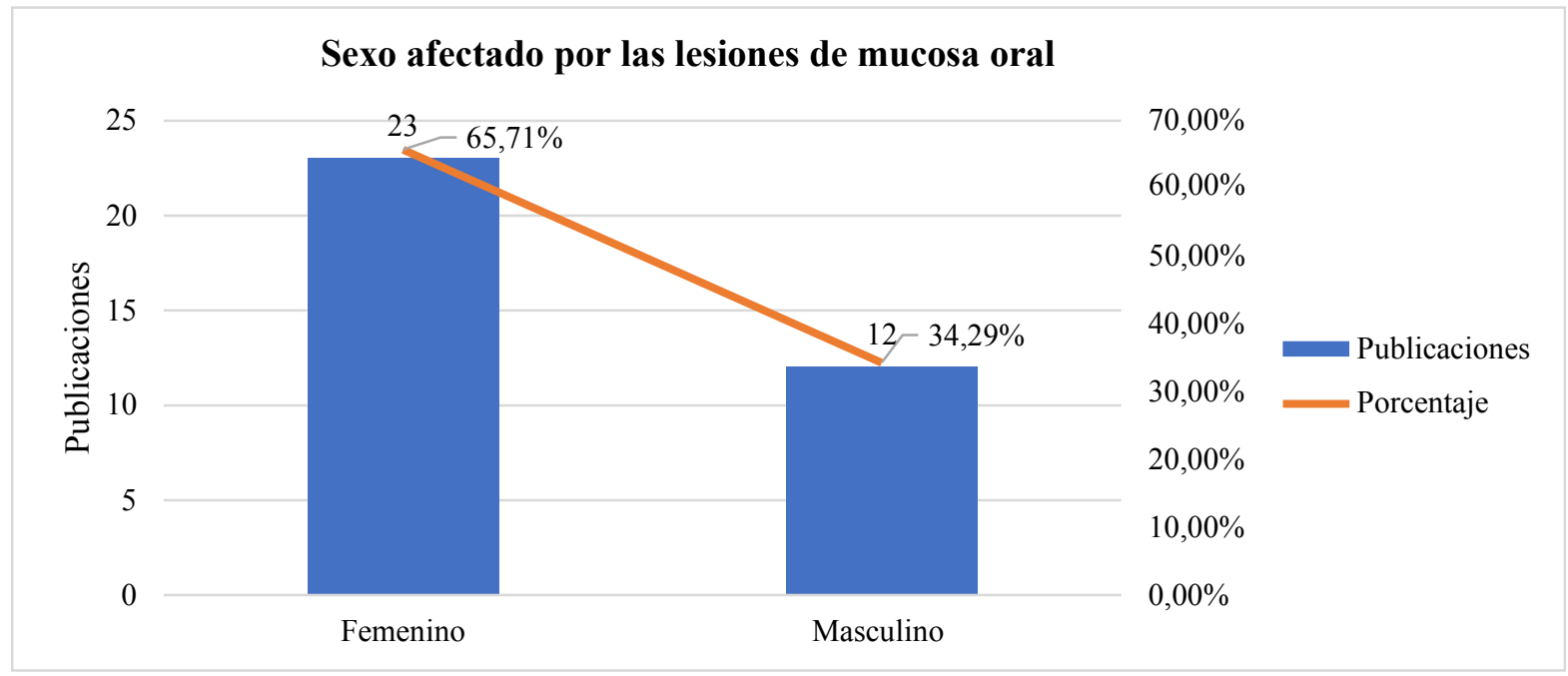

\section{- Edad de los edéntulos totales afectados por las lesiones de la mucosa oral}

Como se puede observar en la figura 5, los más afectados por las lesiones de la mucosa oral asociadas al uso de la prótesis dental en pacientes edéntulos totales son los pacientes mayores de 70 
años con el 22,86\%. Turker (13), aducen que todas las lesiones relacionadas con la dentadura aumentan con la edad del paciente y la duración del uso de la prótesis. (9)

Marín (24) y Mubarak (26), mencionan que con el avance de la edad, los seres humanos envejecen manifestando características de desgaste dentario, pérdida de piezas dentales, grado de oscurecimiento, disminuye la altura ósea alveolar, atrofias de la mucosa oral, además de cambios secundarios, que provoca disminución de la secreción salival, variación de la composición salival por efecto de consumo de medicamentos, que incide directamente en la lesiones de la mucosa oral con influencia de la prótesis completa. (13)

Figura 5. Caracterización de los pacientes con lesiones de la mucosa oral por efecto de la prótesis

\section{por edad}

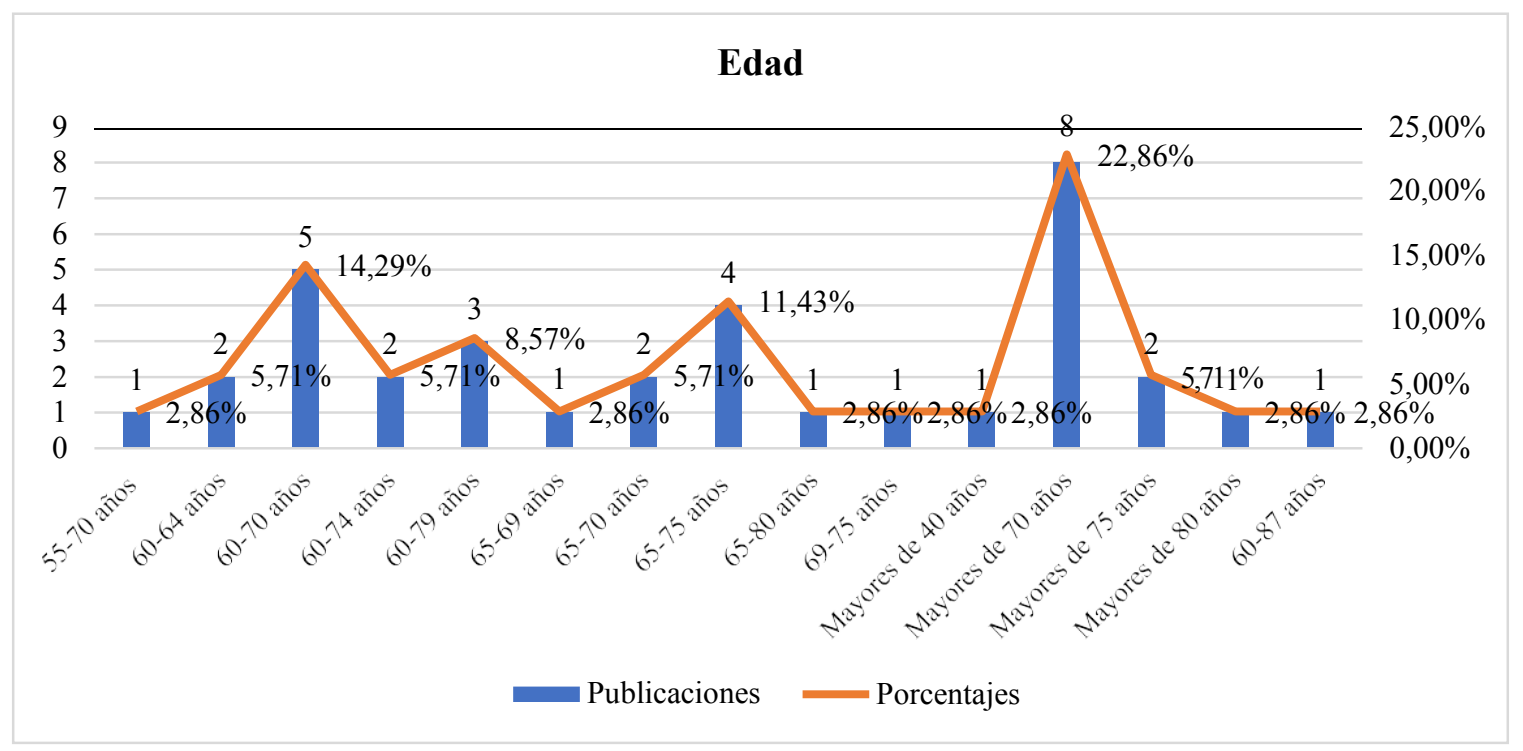




\section{Conclusiones.}

Mediante la revisión bibliográfica se determinó que las principales lesiones de la mucosa oral asociado al uso de la prótesis que se reportaron en la investigación son la estomatitis protésica $(71,43 \%)$, el segundo tipo de lesión es úlcera traumática $(28,57 \%)$, y el tercer tipo de lesión es la Hiperplasia fibrosa $(54,29 \%)$, las primeras causas de las lesiones de la mucosa oral asociado al uso de la prótesis en pacientes edéntulos totales, son por uso prolongado de la prótesis $(22,86 \%)$. Los edéntulos totales también reportan afectación en el daño en el paladar duro $(57,14 \%)$, dolor (7,14\%), inflamación $(7,14 \%)$ y lesiones pre malignas $(7,14 \%)$ como consecuencias del uso excesivo de la prótesis. Los edéntulos totales que son más propenso a presentar las lesiones de la mucosa oral asociado al uso de la prótesis son de sexo femenino (65,71\%), y el masculino con el (34,29\%) con una edad avanzada (mayores de 70 años de edad que representa el 22,86\%). Los pacientes con edad entre 60 y 70 años son los más afectados por estomatitis protésica $(14,29 \%)$.

Conflicto de intereses: los autores declaran que no existe conflicto de intereses.

\section{Bibliografía.}

1. Joya L, Quintero L. Caracterización de la salud bucal de las personas mayores de 65 años de la ciudad de Manizales, 2008. Hacia promoc salud [Internet]. 2015;20(1):140-52. Available from: http://www.scielo.org.co/pdf/hpsal/v20n1/v20n1a10.pdf

2. El Toumb S, Cassia A, Bouchi N, Kassab I. Prevalence and Distribution of Oral Mucosal Lesions by Sex and Age Categories: A Retrospective Study of Patients Attending Lebanese School of Dentistry. Int J Dent. 2018;(4030134):1-6.

3. Gutiérrez M, Sánchez Y, Castillo Y. Lesiones en la mucosa oral de pacientes mayores de 60 años y portadores de prótesis. Correo Científico Médico [Internet]. 2013;17(4):452-60. Available from: http://scielo.sld.cu/scielo.php?script=sci_arttext\&pid=S156043812013000400005

4. Oliveira, Andreza; Carvalho, Helene; Tramontani, Bruna; Sayed, Thays; Santos, Livia; Oliveira, Geraldo; Cantisano M. Prevalence of oral lesions caused by removable. Rev Bras 
Odontol [Internet]. 2016;73(2):130-4. Available from: https://www.researchgate.net/profile/Picciani_BLS/publication/305216698_Prevalence_of_or al_lesions_caused_by_removable_prosthetics/links/578d078808ae59aa66815367/Prevalenceof-oral-lesions-caused-by-removable-prosthetics.pdf

5. Van der Waal I. Potentially malignant disorders of the oral and oropharyngeal mucosa; terminology, classification and present concepts of management. Oral Oncol [Internet]. 2009;45(4-5):317-23. Available from: https://www.ncbi.nlm.nih.gov/pubmed/18674954

6. Zissis A, Yannikakis S, Harrison A. Comparison of denture stomatitis prevalence in 2 population groups. Int J Prosthodont [Internet]. 2006;19(6):621-5. Available from: https://www.ncbi.nlm.nih.gov/pubmed/17165305

7. Espinosa L. Alteraciones de la mucosa bucal, en paciente geriátricos portadores de prótesis dental, en el programa 60 y piquito Sector Cuerpo Quito Sur, Ejército de Salvación, Distrito Metropolitano de Quito. Universidad Central del Ecuador; 2014.

8. Mayorga D. Incidencia de las principales patologías orales en pacientes geriátricos del hogar de ancianos Instituto Estupiñan de Latacunga. Universidad Nacional de Chimborazo; 2017.

9. Otero E, Peñamaría M, Rodríguez M, Martín B, Blanco A. Candidiasis oral en el paciente mayor. Av Odontoestomatol [Internet]. 2015;31(3):135-48. Available from: http://scielo.isciii.es/scielo.php?script=sci_arttext\&pid=S0213-12852015000300004

10. Ercalik S, Ozcan M. Association between Oral Mucosal Lesions and Hygiene Habits in a Population of Removable Prosthesis Wearers. J Prosthodont [Internet]. 2014;17:1-8. Available from: https://onlinelibrary.wiley.com/doi/pdf/10.1111/jopr.12208

11. Behar D. Metodología de la Investigación. Shalom, editor. Madrid; 2008. 94 p.

12. Ali M, Joseph B, Sundaram D. Prevalence of oral mucosal lesions in patients of the Kuwait University Dental Center. Saudi Dent J [Internet]. 2013;25(3):111-8. Available from: https://www.ncbi.nlm.nih.gov/pmc/articles/PMC3809497/

13. Turker S, Sener D, Koc ak A, Yılmaz S, Kulak Y. Factors triggering the oral mucosal lesions by complete dentures. Arch Gerontol Geriatr [Internet]. 2010;51(2):100-104. Available from: https://www.sciencedirect.com/science/article/pii/S0167494309002015?via\%3Dihub

14. Lemus L, Triana K, Selenenko O, Fuertes L, Sáez R. Rehabilitaciones protésicas y su calidad como factor de riesgo en la aparición de lesiones en la mucosa bucal. Rev Cubana Estomatol [Internet]. 2009;46(1):1-14. Available from: http://scielo.sld.cu/scielo.php?script=sci_arttext\&pid=S0034-75072009000100003

15. Shah A, Ahmad T. Oral mucosal lesions in complete denture wearers. J Pakistan Assoc Dermatologists [Internet]. 2011;21(3):170-3. Available from: https://www.researchgate.net/publication/292924671_Oral_mucosal_lesions_in_complete_de nture_wearers

16. Serrano M, Téllez N, Chabot L. Alteraciones clínicas en mucosa bucal de pacientes 
portadores de prótesis totales residenciados en el centro "Dr. Ricardo Sergent" (INASS). Rev Venez Invest Odont IADR. 2015;3(2):111-9.

17. García B, Capote M, Morales J. Prótesis totales y lesiones bucales en adultos mayores institucionalizados. Rev Finlay [Internet]. 2012;2(1):32-44. Available from: http://www.revfinlay.sld.cu/index.php/finlay/article/view/99

18. Zwiri A. he Prevalence And Associated Factors Of Denture Wearing Associated Oral Lesions Among Dental Patients Attending College Of Dentistry Clinics In Aljouf University. Eur Sci J [Internet]. 2016;12(9):326-32. Available from: http://eujournal.org/index.php/esj/article/view/7201

19. Gaur A, Kumar G, Siddiqui S, Agarwal S, Singh Monga H, Gosavi S. Study of Prevalence of Oral Lesions in Complete Denture Wearers. J Int Oral Heal [Internet]. 2015;7(11):97-100. Available from: http://www.ispcd.org/userfiles/rishabh/V7I11/V7I11A21.pdf

20. Matos G, Andrade P, Rode A, Araújo C, Almeida V. Prevalencia de lesiones bucales asociadas al uso de prótesis dentales removibles, en un servicio de estomatología. Rev Cubana Estomatol [Internet]. 2011;48(3):268-76. Available from: http://scielo.sld.cu/scielo.php?script=sci_arttext\&pid=S0034-75072011000300008

21. Gendreau L, Loewy Z. Epidemiology and etiology of denture stomatitis. J Prosthodont [Internet]. 2011;20(4):251-60. https://www.ncbi.nlm.nih.gov/pubmed/21463383

22. Atashrazm P, Sadri D. Prevalence of Oral Mucosal Lesions in a Group of Iranian Dependent Elderly Complete Denture Wearers. J Contemp Dent Pract [Internet]. 2013;14(2):174-8. Available from: https://www.researchgate.net/publication/243967784/download

23. Bomfim I, Soares D, Tavares G, Santos R, Araújo T, Padilha W. Prevalência de Lesões de Mucosa Bucal em Pacientes Portadores de Prótese Dentária. Pesqui Bras Odontopediatria Clin Integr [Internet]. 2008;8(1):117-21. Available from: http://www.redalyc.org/articulo.oa?id=63711702019

24. Marín W, Veiga L, Reyes Y, Mesa D. Lesiones bucales en adultos mayores y factores de riesgo. Policlínico "Dr. Tomás Romay", La Habana, Cuba. La Habana, Cuba Rev haban Cienc médica.

25. Dantas de Medeiros, Fabianna; De Araújo-Silva, Thiago; Alves Ferreira, Kleiton; OliveiraMoura, Jamile; Cavalcanti-Lima, Isabela; Guerra-Seabra E. Uso de prótese dentária e sua relação com lesões bucais. Rev salud pública [Internet]. 2015;17(4):603-11. Available from: Uso de prótese dentária e sua relação com lesões bucais

26. Mubarak S, Hmud A, Chandrasekharan S, Ali A. Prevalence of denture-related oral lesions among patients attending College of Dentistry, University of Dammam: A clinicopathological study. J Int Soc Prev Community Dent [Internet]. 2015;5(6):506-512. Available from: http://www.jispcd.org/article.asp?issn=22310762 ; year $=2015 ;$ volume $=5 ;$ issue $=6$; spage $=506$; epage $=512$; aulast $=$ Mubarak 
27. Nápoles I, Rivero O, García C, Pérez D. Lesiones de la mucosa bucal asociadas al uso de prótesis totales en pacientes geriatras. Arch Médico Camagüey [Internet]. 2016;20(2):158-66. Available from: http://scielo.sld.cu/scielo.php?script=sci_arttext\&pid=S102502552016000200008

28. Mandali G, Sener D, Turker S, Lgen HU. Factors affecting the distribution and prevalence of oral mucosal lesions in complete denture wearers. Gerodontology [Internet]. 2011;28(2):97103. Available from: https://www.ncbi.nlm.nih.gov/pubmed/20579108

29. Lira A, Orestes A, Oliveira M, Orestes M. Lesões da mucosa oral em pacientes portadores de próteses dentárias: ilustrações clínicas e abordagem preventiva. Rev Odonto [Internet]. 2008;16(31):19-36. Available from: https://www.metodista.br/revistas/revistasims/index.php/Odonto/article/view/601/599

30. Gheno J, Martins M, Munerato M, Hugo F, Sant'Ana F, Weissheimer C, et al. Oral mucosal lesions and their association with sociodemographic, behavioral, and health status factors. Braz Oral Res [Internet]. 2015;29(6):1-6. Available from: https://www.lume.ufrgs.br/bitstream/handle/10183/130245/000977315.pdf?sequence=1

31. Carnevale S, Bassan N, Roy O. Detección de lesiones bucales en una población geriátrica. Actual odontológicas GADOR [Internet]. 2009;50(1):1-8. Available from: http://www.gadornet.com.ar/iyd/fao/fao50.pdf

32. Fantin M, García H. Factores familiares, su influencia en el consumo de sustancias adictivas. Lib Rev Psicol [Internet]. 2011;21(1):141-51. Available from: http://www.redalyc.org/pdf/686/68639580013.pdf

33. Moreno J, Montoya A, Gómez D, Arboleda A, Zea F, Agudelo A. Situación de salud bucal y estado protésico del paciente edentado bimaxilar que acude a la Facultad de Odontología de la Universidad de Antioquia: un estudio piloto. Rev Fac Odontol Univ Antioq [Internet]. 2012;24(1):22-36. Available from: http://aprendeenlinea.udea.edu.co/revistas/index.php/odont/article/view/10400/12194 